\title{
Micro Gasturbine Integrated Design. Part 2: Compressor and Turbine
}

\author{
Dario Barsi, Tiziano Garbarino, Andrea Perrone*, Luca Ratto, Gianluca Ricci, Fabrizio Stefani, Pietro Zunino \\ DIME - University of Genova, Via Montallegro 1, 16145, Genova, Italy
}

\begin{abstract}
Multidisciplinary design optimization (MDO) is widely employed to enhance turbomachinery components efficiency. The aim of this work is to describe a complete tool for the aero-mechanical design of a radial inflow turbine and a centrifugal compressor. The high rotational speed of such machines and the high exhaust gas temperature (for the turbine) exposes blades to really high stresses and therefore the aerodynamics design has to be coupled with the mechanical one through an integrated procedure. This approach employs a fully 3D Reynolds Averaged Navier-Stokes (RANS) solver for the aerodynamics and an open source Finite Element Analysis ( ) solver for the mechanical integrity assessment.Due to the high computational cost of both these two solvers, a metamodel, such as an artificial neural network, is employed to speed up the process. The interaction between two codes, the mesh generation and the post processing of the results are achieved via in-house developed scripting modules. The obtained results are widely presented and discussed.
\end{abstract}

Keywords: Cogeneration, Centrifugal Compressor, Radial Turbine, MicroGT

\section{Introduction}

Compressor and turbine optimized design is akey element for enhancing the overall efficiency of micro gasturbines. In particular, components efficiency heavily affects plant performance and aerodynamic design of novel geometries has to consider mechanical constraints in order to pursue this target without compromising machine integrity [1],[2].

The aerodynamic design carried out through CFD optimization techniques leads to the definition of a proper geometry configuration able to fulfill the requested aerodynamic performance but, at the same time, geometrical parameters modification necessarily involves structural and mechanical aspects that have to be considered and taken into account.

Nowadays, next steps of improvements are based on multidisciplinary optimization, because the aerodynamic design has to be strongly coupled with mechanical assessments ([1],[2],[3]). In fact, without considering this strong interaction, finding a satisfactory compromise solution would require many design iterations between aerodynamics and mechanical experts.

The proposed optimization procedure is focused on efficiency enhancement guaranteeing a safe turbine and compressor operation, by limiting stresses and avoiding resonances.

A significant and relevant aspect of this work is the linking of aerodynamic design optimization (through commercial codes) and mechanical optimization through opensource software.

\section{Compressor}

The centrifugal compressor, which the optimization procedure has been applied to, has been designed starting from a one-dimensional preliminary approach aimed at

\footnotetext{
${ }^{*}$ Corresponding author

Email: andrea.perrone@edu.unige.it

(c) 2016 International Association for Sharing Knowledge and Sustainability

DOI: $10.5383 /$ ijtee.11.01.003
} 
fulfilling the design duty with the best machine efficiency, taking also into account mechanical limitations of the materials. In this first design step the main dimensions and blade angles have been found applying 1D equations, starting from empirical data and statistical analyses to fix the ranges for the main unknown design parameters. For the preliminary design, an in house developed code based on the procedure suggested by Vavra [4], has been adopted, extending the equations applicability to the cases of not purely radial blades at the impeller discharge and of absolute inlet velocities not axially directed.

\section{A. Geometry Parameterization}

The commercial software Numeca/Autoblade [5] has been employed to choose a proper set of parametric curves and to identify the most appropriate geometrical topology that enables designer to manage different geometrical shapes via a limited number of key parameters automatically defined within the optimization environment.

For achieving axial-to-radial configuration both hub and shroud contours present the same geometrical topology: an axial line part, followed by a Bezier curve with 4 control points, to end it up with another line, radially directed.

The impeller row is made up of a main blade and a splitter blade. Impeller main blade is stacked on leading edge (LE). LE meridional projection is a line controlled by extreme points respectively on hub and shroud wall. LE shape in the $\vartheta$-span frontal plane is linear too, controlled by defining its slope and its starting tangential position at hub. The meridional location of main blade is fully set by adding a trailing edge (TE) linear definition. For main blade camber parameterization a Bezier curve, controlled by 5 points equally distributed along the abscissa, has been employed for each of the three spanwise sections; among these control points the first one is automatically positioned by LE location. The suction and pressure sides have been defined by adding a symmetric half-thickness profile normal to camber curve. For half-thickness law definition, a Bezier curve with 4 control points has been employed on each spanwise section. The first control point is rigidly joined to main blade LE while the second one can vary its position in order to control the LE radius amplitude. The third one is located in the middle of camber abscissa and tunes the maximum thickness of profile; the last trims the profile so as to obtain a blunt TE. The camber shape and half-thickness law of splitter are inherited from the corresponding ones of main blade. On each spanwise section splitter profile is tangentially placed in the middle of main blade channel while along meridional direction it starts at an arbitrary position and ends at main blade TE location.
The diffuser row is made up of straight wedge-shaped blades. They are cylindrical, unleaned and not tapered blades, therefore they have been defined only by means of a hub surface profile axially extruded up to intersect shroud surface. For diffuser camber parameterization a line has been chosen. For half-thickness law definition, a Bezier curve with 3 control points has been employed. The third one is placed at diffuser TE location and controls the maximum thickness of blunt profile. After imposing the required constraints the parametric geometrical model has 26 degrees of freedom.

\section{B. Fluid and Solid Mesh Features, Boundary Conditions}

The whole fluid domain has been meshed by means of Numeca/IGG Autogrid5 [5]. A python script that automatically manages the multirow configuration has been implemented in order to simplify the interfacing with the optimization environment. For each row involved a multi-block hexahedral structured mesh is built. The entire mesh is made up of about 2 million of cells: 1.35 million in the impeller row, 0.05 million in the inlet duct and 0.6 million in the diffuser row. The CFD calculation is arranged throughout Numeca/FineTurbo [5] and is carried out by means of Numeca/Euranus Navier Stokes equations solver [6]. A $R A N S$ steady approach is followed and the two equations SST model has been chosen for representing turbulence phenomenology. The interface between impeller and diffuser row is managed by mixing plane approach. Absolute total flow conditions, absolute flow angles, turbulent kinetic energy and turbulent dissipation rate values determined by empirical relations are set at inlet while mass flow rate is imposed at outlet. The solid mesh has been created by the open source software GMSH [7], integrated in an in house developed Python procedure.

\section{Optimization Process Settings}

For the centrifugal compressor database generation, due to the high number of variables involved in the optimization process, a $25 \mathrm{X}$ random sampling has been chosen. In fact, other more refined DoE techniques would lead to too expensive computational effort. Giving to each parameter the possibility to assume nine different values between its lower and upper bound, 700 CFD calculations, chosen randomly among the available ones, have been performed as database samples.

The optimization procedure is applied in order to minimize a single objective function that is composed by the sum of several dimensionless penalty terms (Equation 1), each of which contains a structural or aerodynamic quantity. Due to the parabolic form of each penalty term the objective function is convex, therefore a minimum always exists. Each parabolic penalty term has a weighting coefficient. In the centrifugal compressor optimization six penalty terms are employed, three aerodynamic (Equation 2 - 4), three structural (Equation $5-7)$. 


$$
\begin{aligned}
& P_{\text {tot }}=P_{\eta_{\text {istt }}}+P_{\beta_{\text {tt }}}+P_{M a_{c}}+P_{\text {stress }}+P_{\text {freq_low }}+P_{\text {frep_high }} \\
& P_{\eta_{i s t t}}=\left\{\begin{array}{c}
W_{\eta_{i s t t}}\left(\frac{1-\eta_{i s t t}}{0.1}\right)^{2} \quad \text { if } \eta_{\text {istt }}<1 \\
0 \quad \text { if } \eta_{\text {istt }} \geq 1
\end{array}\right. \\
& P_{\beta_{t t}}=\left\{\begin{array}{c}
W_{\beta_{t t}}\left(\frac{4.5-\beta_{t t}}{1}\right)^{2} \quad \text { if } \beta_{t t}<4.5 \\
0 \quad \text { if } \beta_{t t} \geq 4.5
\end{array}\right. \\
& P_{M a_{c}}=\left\{\begin{array}{cc}
W_{M a_{c}}\left(\frac{1-M a_{c}}{1}\right)^{2} & \text { if } M a_{c} \geq 1 \\
0 & \text { if } M a_{c}<1
\end{array}\right. \\
& P_{\text {stress }}=\left\{\begin{array}{c}
W_{\text {stress }}\left(\frac{\sigma_{\max }}{\sigma_{\text {all }}}\right)^{2} \quad \text { if } \sigma_{\text {max }} \geq \sigma_{\text {all }} \\
0 \quad \text { if } \sigma_{\text {max }}<\sigma_{\text {all }}
\end{array}\right. \\
& \sigma_{\text {all }}=\frac{\sigma_{y}(\text { yielding })}{S F(\text { safety factor })} \\
& P_{\text {freq_low }}=\left\{\begin{array}{cl}
W_{\text {freq_low }}\left(\left|\frac{f_{\text {eigfr }}}{f_{\text {exc_low }}}-1\right|\right)^{2} & \text { if }\left|\frac{f_{\text {eigfr }}}{f_{\text {exc_low }}}-1\right| \leq 0.05 \\
0 & \text { if }\left|\frac{f_{\text {eigfr }}}{f_{\text {exc_low }}}-1\right|>0.05
\end{array}\right. \\
& P_{\text {freq__high }}=\left\{\begin{array}{cc}
W_{\text {freq__high }}\left(\left|\frac{f_{\text {eigfr }}}{f_{\text {exc_high }}}-1\right|\right)^{2} \text { if }\left|\frac{f_{\text {eigfr }}}{f_{\text {exc_high }}}-1\right| \leq 0.05 \\
0 & \text { if }\left|\frac{f_{\text {eigrr }}}{f_{\text {exc_high }}}-1\right|>0.05
\end{array}\right.
\end{aligned}
$$

The objective function is composed, regarding to aerodynamic aspects, in order to maximize the total-tototal isentropic efficiency, to guarantee the design absolute total pressure ratio and an absolute subsonic flow condition at diffuser inlet. For what concerns the structural behavior, the aim is to keep the maximum Von Mises stress in the impeller lower than the allowable stress value and to avoid the rotor resonances.

\section{Aerodynamic Optimization Results}

The optimization process has reached convergence after 35 iterations. The optimized configuration is shown in this paragraph highlighting the differences with respect to the original one and the effects of the new geometrical arrangement on the aerodynamic and structural behavior. The physical responses to the objective function imposed are therefore explained.

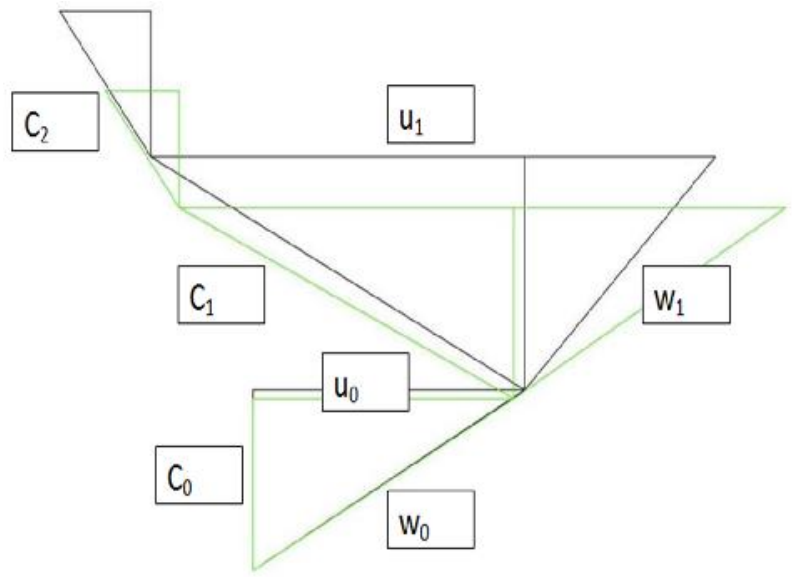

Figure 1 - Velocity triangles for Original (black) and Optimized (green) Configuration at Midspan Section

Figure 2 shows that starting from impeller inlet section relative Mach number maximum value is decreased from 1.35 to 1.2 in the optimized configuration; moreover the region characterized by relative supersonic flow has been significantly reduced. Relative Mach number decreasing is mainly due to the lower inlet meridional velocity $\left(\mathrm{c}_{0}\right.$ in Figure 1) obtained by means of inlet area widening (Figure 4). As concerns BtB geometrical parameters, the reduced blade thickness weakens the shock wave strength. However is the local null value of camber curvature at LE that provides the greater contribution to shock wave reduction.

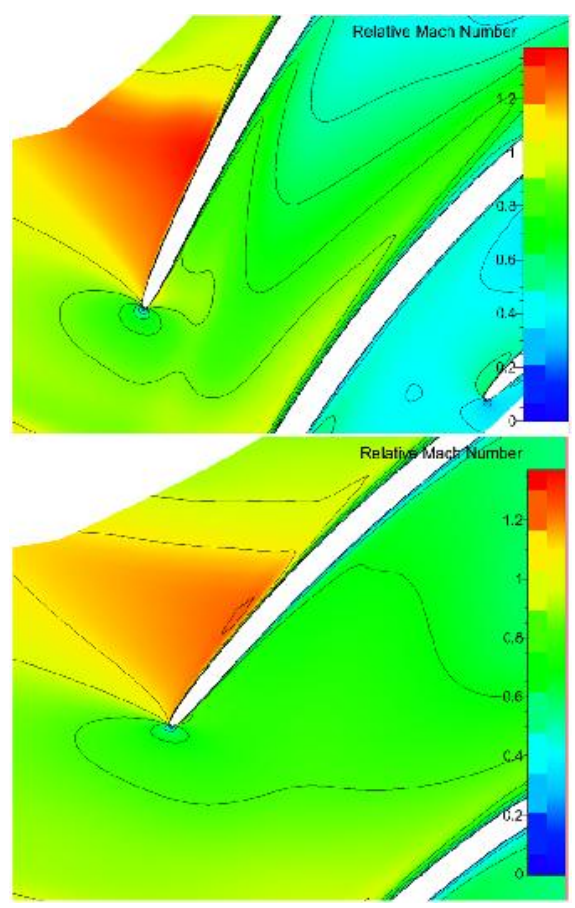

Figure 2 - Relative Mach Number Field for Original (top) and Optimized (bottom) Configuration on the Tip BtB Surface 
With respect to the original configuration the flow, once passed through the optimized impeller blade, undergoes a strong increment of the exit relative flow angle (Figure 1) since the metal backswept angle changes from $30^{\circ}$ to $50^{\circ}$ and the slip factor decreases. However the velocity ratio $\mathrm{w}_{0} / \mathrm{w}_{1}$ isn't affected by a significant decrease since impeller outlet area widening enables meridional velocity reduction.
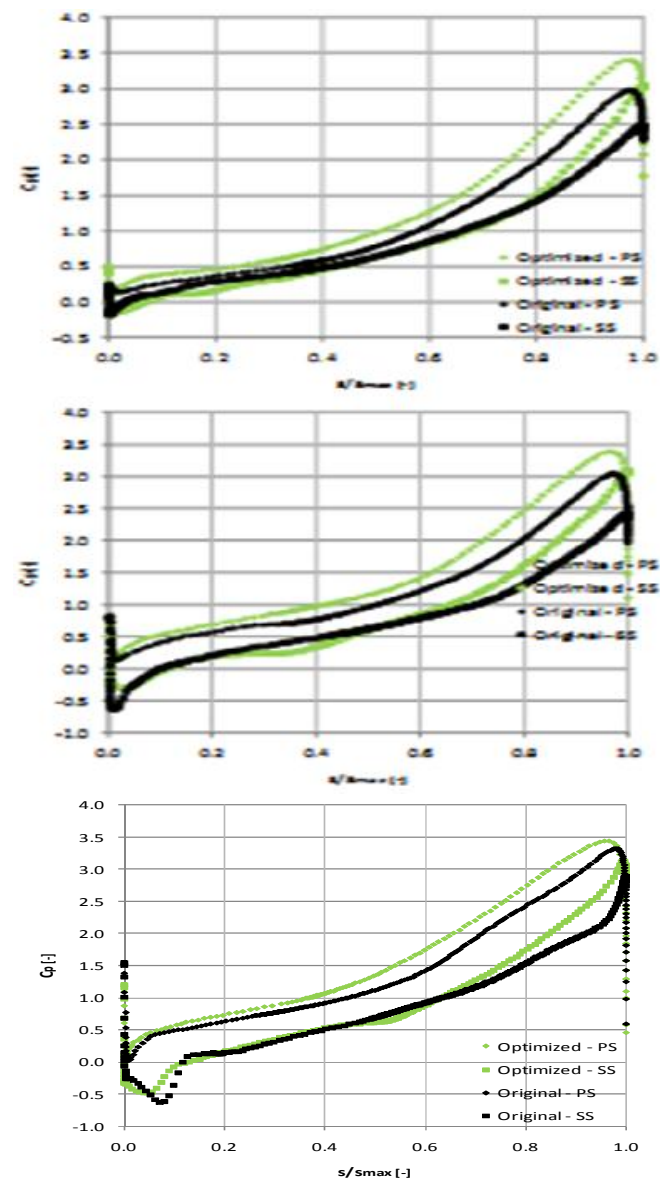

Figure 3 - Pressure Coefficient Diagrams for Impeller Main Blade Original (black) and Optimized (green)

Configuration at Hub (top-left), Midspan (top-right) and Tip (bottom) Sections

From the pressure coefficient diagrams (Figure 3) it can be noted that in the optimized configuration the blade loading has been increased for all the considered sections, with particular improvements for the hub one, which presents the worst blade loading in the original configuration. This behavior is consistent if it is observed that similar absolute total pressure ratio is achieved with 22 instead of 24 blades.

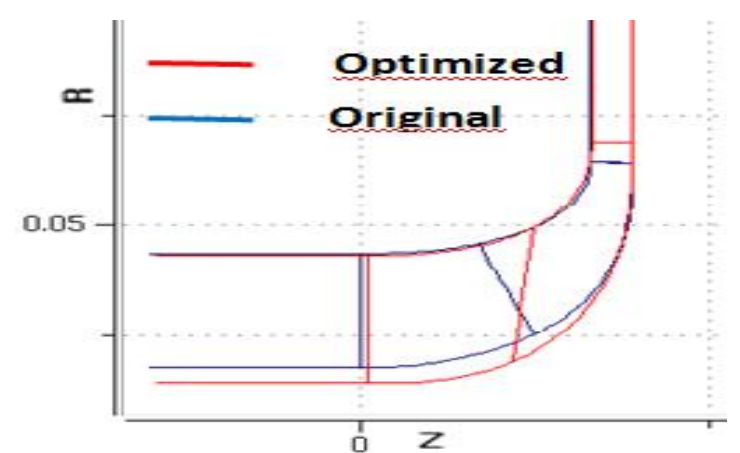

Figure 4 - Meridional Section of the Original (blue) and Optimized (red) Configuration

Figure 5 shows that in the optimized configuration the maximum value of absolute Mach number decreases to 0.98 , hence resulting a subsonic flow regime at diffuser inlet. This behaviour is achieved by reducing the absolute velocity magnitude at impeller exit (Figure 5) thanks to an increase both of the backswept angle (that leads to a higher exit relative flow angle) and of the impeller trailing edge radial position (causing a meridional velocity fall down that fully counterbalances the peripheral velocity increment).

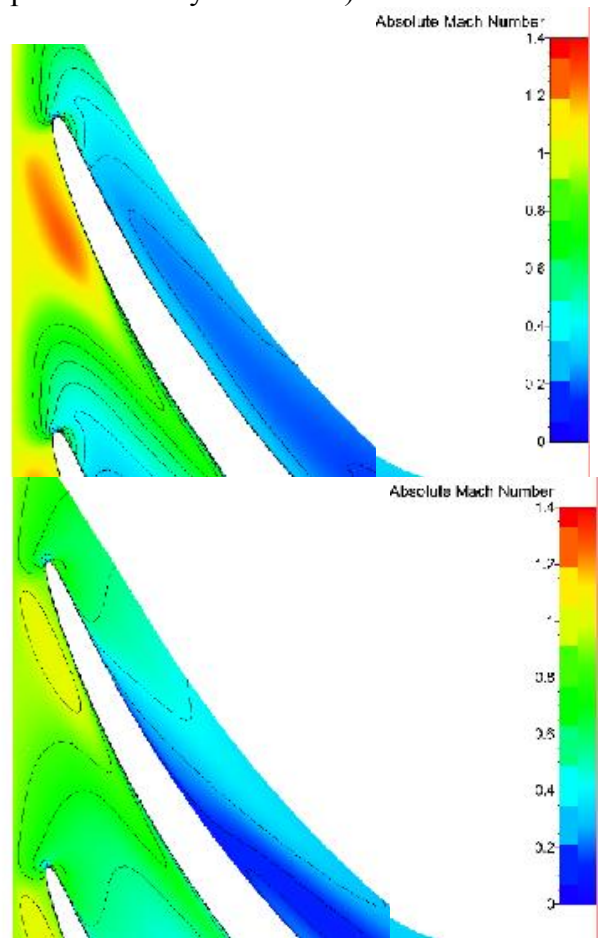

Figure 5 - Absolute Mach Number Field for Original (top) and Optimized (bottom) Configuration on the Midspan BtB Surface

Concerning the diffuser row, Figure 6 shows a smoother load distribution for the optimized configuration. Moreover, it's worth noting that the increase of static pressure is performed up to the trailing edge, while the original diffuser presents an unloaded zone in the rear part of the blade. 


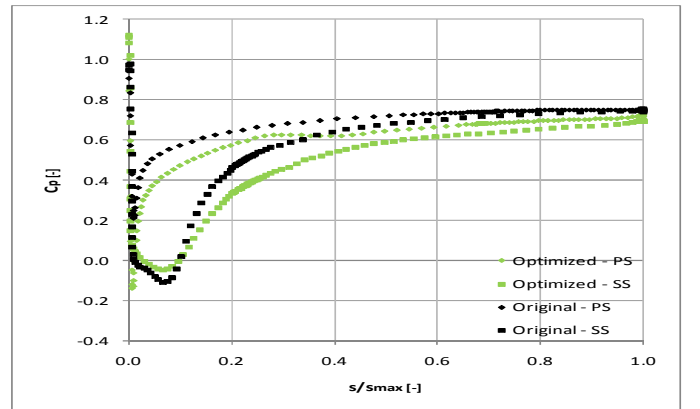

Figure 6 - Pressure Coefficient Diagram for Diffuser Blade Original (black) and Optimized (green) Configuration at Midspan Section

\section{E. Structural Optimization Results}

For what concerns the mechanical integrity assessments, the original and the optimized configuration show similar maximum Von Mises stress (Figure 7). The FEM calculations have been carried out through the open source software Calculix [8]. However, in the optimized configuration the Von Mises stress map is smoother and the stress at the hub has been reduced.
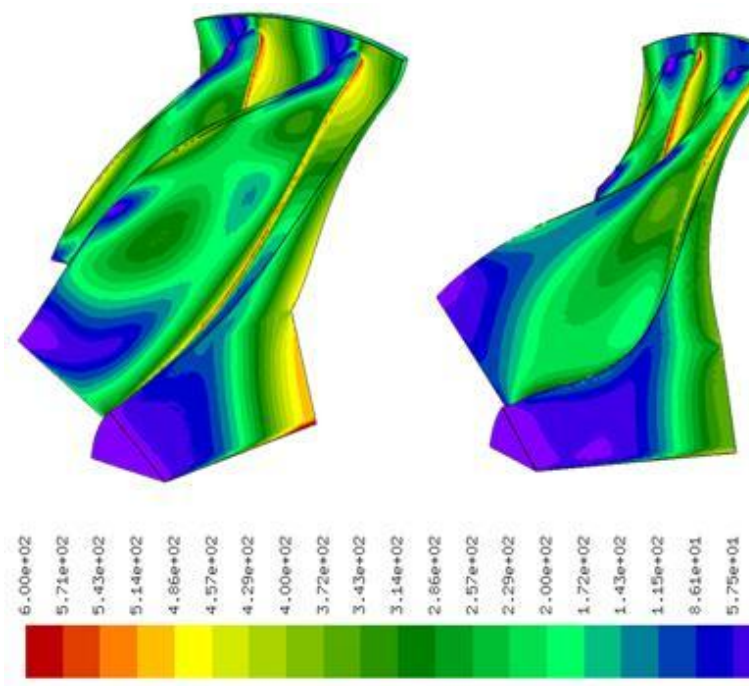

Figure 7 - Von Mises Non Dimensional Stress for the Original and the Optimized Configuration

In the original impeller the maximum displacements (1.1 $\mathrm{mm})$ is located at the tip of the splitter. In the optimized configuration the maximum displacement is $0.37 \mathrm{~mm}$.

The original configuration presents resonances with the first $4 \mathrm{EO}$ and with the number of stators downstream the impeller; in the optimized one, the optimization process has modified the blade design in order to meet all the dynamic assessment criteria.

The original and optimized performances are summarized in Table 1.
Table 1: Original and Optimized Performances for Centrifugal Compressor

\begin{tabular}{c||c|c} 
& Original & Optimized \\
\hline \hline$\dot{m}[\mathrm{~kg} / \mathrm{s}]$ & 0.74 & 0.74 \\
\hline$\eta_{\text {ist }-t}[-]$ & 0.80 & 0.82 \\
\hline$\beta_{t-t}[-]$ & 4.3 & 4.4 \\
\hline Inlet relative Mach at impeller tip [-] & 1.35 & 1.2 \\
\hline Absolute Mach at diffuser inlet [-] & 1.4 & 0.98 \\
\hline Stress usage factor $[-]$ & 1.2 & 0.95 \\
\hline Resonance 4EO & Yes & No \\
\hline Resonance NPF & Yes & No \\
\hline
\end{tabular}

\section{Turbine}

The preliminary design of the whole machine has been determined through a literature review from more than 20 papers presented in the last ten years in International Conference Proceedings and Journals.

\section{A. Geometry Parameterization}

The three-dimensional representation of the machine is defined by the meridional channel geometry, the stator and rotor blade camber line and thickness curves and by the definition of blade positions along the channel (Figure 8).

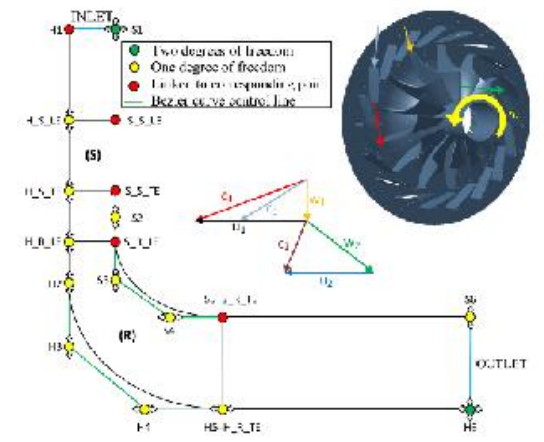

Figure 8: Turbine Geometrical Parameterization and Velocity Standards

Similarly to compressor parameterization, Bezier curves have been used for geometry representation within the automatic optimization tool.

The stator row has been defined by employing a simple Bezier for the camber line, while the thickness law is defined as thickness addition on the camber curve with a simple two parameter law. The rotor row has been defined by employing a Bezier curve with four control points for both camber line and thickness law. In conclusion, the total number of design parameters, which will be managed by the optimization procedure, is 22 .

\section{B. Fluid And Solid Mesh Features, Boundary Conditions}

Once the geometry has been defined, the fluid domain has been meshed. For both stator and rotor blade a 
Numeca Autogrid[5]default topology has been chosen. The stator row has been meshed with about 500.000 grid nodes, while the rotor row requires about one million of nodes. The solid mesh procedure is similar to that one described in the compressor section.

\section{Optimization Process Settings}

The developed procedure combines the aerodynamic optimization algorithm implemented in Fine/Design3D [5]with CalculiX[8], a free open source FEA.

Every sample analysed through CFD for evaluating its aerodynamic performance is also verified for its structural behaviour.

From the aerodynamic optimization point of view, each CFD calculation is carried out by imposing total conditions and flow angle at the inlet section and averaged static pressure at the outlet section. The effects of turbulence has been considered by means of $\mathrm{k} \omega$-SST turbulence model.

Once the proper geometrical constraints have been set, a $15 \mathrm{X}$ random sample has been chosen for database generation. Each parameter has been split in five different values, and about 350 sample CFD calculations, chosen with a pseudo-random technique called "random among discrete levels", have been employed. Each computation is run in serial (2.5 hours on modern $\mathrm{CPU}$ ), but the overall database generation has been split over several CPUs.

The objective function for turbine geometry optimization process, to be minimized, is made up of several penalty terms:

$P_{\text {Aero }}=P_{\dot{m}}+P_{M A X \eta_{t-t}}+\sum_{i=1}^{4} P_{M A X \alpha_{O U T, i}}+\sum_{i=1}^{4} P_{M I N \alpha_{O U T, i}}$

where

$$
\begin{aligned}
& P_{\dot{m}}=W_{\dot{m}}\left(\frac{\dot{m}_{\text {nom }}-\dot{m}}{\dot{m}_{\text {nom }}}\right)^{2} \\
& P_{\text {MIN } \alpha_{\text {OUT }, i}}=\left\{\begin{array}{ccc}
W_{\alpha \text { OUT }, i}\left(\frac{\alpha_{\text {OUTMIN }, i}-\alpha_{\text {OUT }, i}}{\alpha_{\text {OUTMIN }, i}}\right) & \text { if } & \alpha_{\text {OUT }, i}<\alpha_{\text {OUTMIN }, i} \\
0 & \text { if } & \alpha_{\text {OUT }, i} \geq \alpha_{\text {OUTMIN }, i}
\end{array}\right. \\
& P_{\text {MAX } \alpha_{\text {OUT }, i}}=\left\{\begin{array}{lll}
0 & \text { if } & \alpha_{\text {OUT }, i}<\alpha_{\text {OUTMAX }, i} \\
W_{\alpha O U T, i}\left(\frac{\alpha_{\text {OUTMAX }, i}-\alpha_{\text {OUT }, i}}{\alpha_{\text {OUTMAX }, i}}\right) & \text { if } & \alpha_{\text {OUT }, i} \geq \alpha_{\text {OUTMAX }, i}
\end{array}\right. \\
& P_{\text {MAXף }_{t-t}}=W_{\eta_{t-t}\left(1-\eta_{t-t}\right)^{2}}
\end{aligned}
$$

The aim of this function is to drive the optimization process to ensure a mass flow rate equal to design condition (Equation (9)), to keep the outlet tangential velocity in a desired range along spanwise direction (sampling the values on four spanwise sections, (Equations (10)-(11)) and to maximize the overall total to total efficiency (Equation (12)).Each term is made non dimensional by using suited reference values.

For a preliminary assessment an allowable stress has been defined, based on yield strength, expressed as a function of temperature, according to the following expression:

$$
\sigma_{\text {all }}=\frac{a_{1} T^{6}+a_{2} T^{5}+a_{3} T^{4}+a_{4} T^{3}+a_{5} T^{2}+a_{6} T+a_{7}}{S F}
$$

where SF is a safety factor and $a_{i}$ properly defined constants. Then, Von Mises stresses of each blade node have been compared to the allowable ones and, since another target is to avoid that Eigen frequencies are in the range $0.95-1.05$ of the exciting frequencies, properly penalty function, similar to the ones defined for the compressor case, have been defined.

Thus, the structural analysis objective function can be obtained as the sum of the previously described terms:

$$
P_{\text {Struct }}=P_{\text {Stress }}+P_{\text {freq_low }}+P_{\text {freq_high }}
$$

\section{Aerodynamic Optimization Results}

Once the aerodynamics and structural objective function have been chosen, the optimization procedure will find the optimum solution for the overall objective function defined as:

$$
P_{\text {tot }}=P_{\text {Aero }}+P_{\text {Struct }}
$$

The optimization process has been set to 30 iterations. In order to understand the phenomena driving the optimization procedure previously discussed, the blade loading distributions in the form of static pressure coefficient $c_{p}$ at hub, midspan, and tip sections are reported in Figure 9 for both the original and the optimized geometries. 


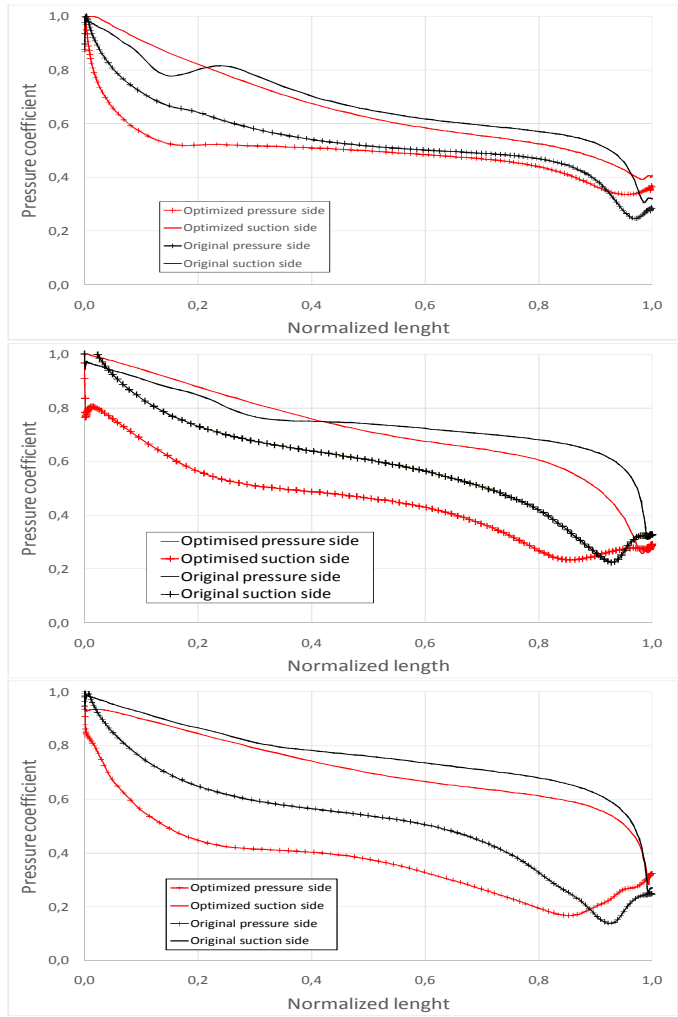

Figure 9 - Pressure Coefficient Diagram for Rotor Original and Optimized Configuration at Hub, Midspan and Tip Sections

We can focus on midspan section to highlight some considerations. For this section also the velocity diagrams for baseline and optimized geometry are reported in Figure 10.

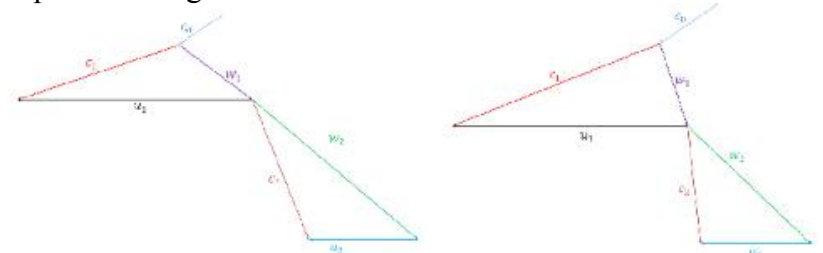

Figure 10 - Velocity Diagram for Original (left) and Optimized (right) Configuration at Midspan

For the original configuration (black curves in Figure 9) the pressure and the suction side lines intersect just behind the impeller leading edge, suggesting the occurrence of a pressure side boundary layer separation due to excessive negative incidence angle condition. The wrong relative inlet flow angle induces the formation of a recirculating flow region.

In the improved configuration the optimization tool increases the tangential component of the absolute velocity at the rotor inlet (reducing the channel height and raising the absolute exit flow angle from the stator blade), thus minimizing the incidence angle at the impeller leading edge. This re-establishes a right incidence condition to the impeller, as shown by the loading distribution in the optimized case (

Figure 12).
As a consequence a higher Mach number at the stator blade trailing edge is obtained for the optimized configuration, as shown in the colour plot of Figure 11, where both the absolute and relative Mach number distributions in the meridional plane are reported.
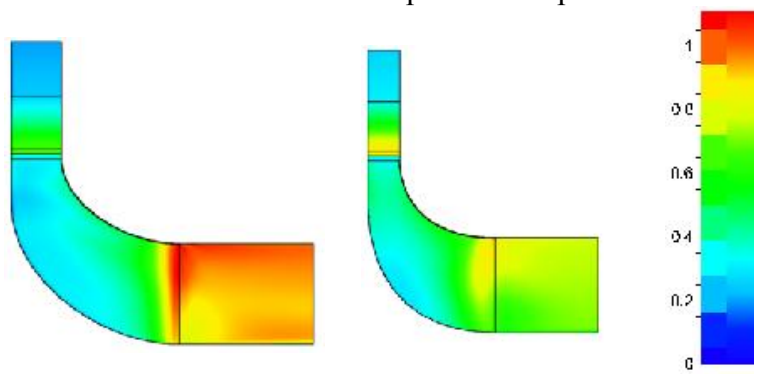

Figure 11: Absolute and Relative Mach Number Distributions in the Meridional Plane: Original (left) and Optimized (right) Configurations

Figure 11clearly highlights flow regions characterized by $M_{\mathrm{r}}>1$. The steep pressure increase induced by the shock wave also causes large viscous losses due to penalties in the suction side boundary layer evolution.

This high relative Mach number characterizing the original case also sets excessive mass flow rate elaborated by the turbine as compared with the target value. Thus, the optimization procedure acts reducing the mass flow rate sensibly modifying the impeller width at the entrance, but keeping the same blade height at the outlet section (see Figure 11). This implies a reduction of the exit relative Mach number, which becomes subsonic in the optimized case.

Both sonic and viscous effects observed in the original case simultaneously contribute to enlarge the entropy production in the rear part of the impeller, as it is make evident by the entropy fields shown in Figure 12.

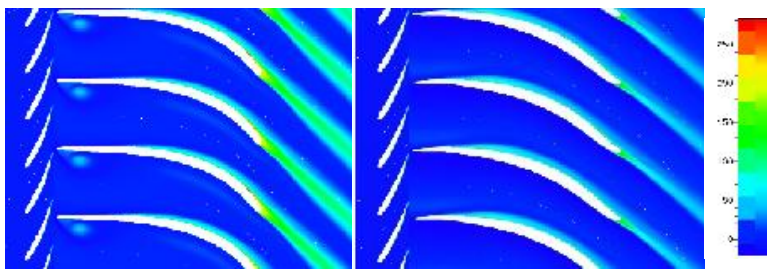

Figure 12: Entropy Field (expressed in J/kgK) for Original (top) and Optimized Configuration (bottom) at Midspan. 


\section{E. Structural Optimization Results}
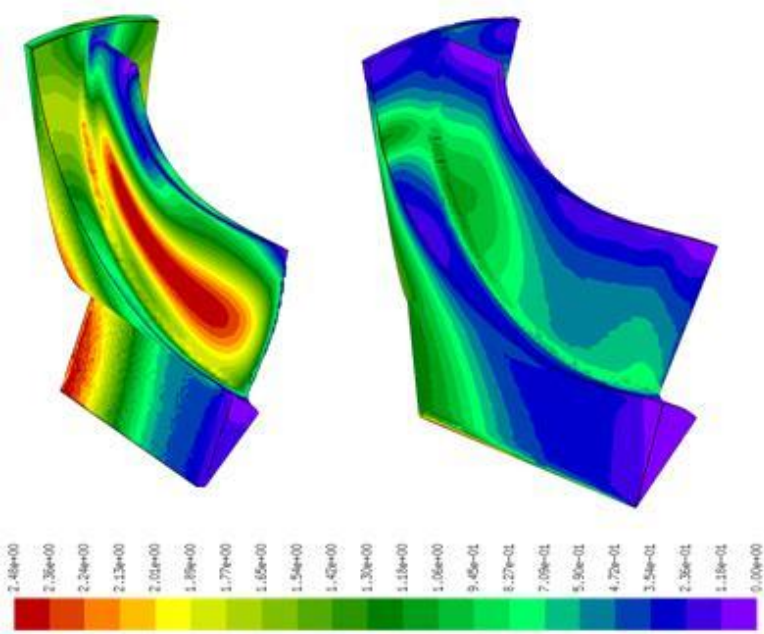

Figure 13: Von Mises Non Dimensional Stress for the Original and the Optimized Configuration

In the original configuration there is a high stress in the central zone of the blade: the lean angle of the blade is very high, so the centrifugal force creates an elevated bending stress across the section. In the optimized configuration the lean angle has been reduced and the higher stress is located in the fillet region, where local plastic strains are allowed: these strains, however, have to be carefully assessed through LCF analysis to predict the life of the impeller.

The bending stress on the blade, consequently, have been significantly reduced.

Figure 14 shows the SAFE (Singh Advanced Frequency Evaluator) diagram for the original impeller, where two resonances are likely to occur in the red circles depicted in the graph. For the final configuration, instead, the optimization tool has modified the blade design, avoiding any possible resonances, both for the first EO and for NPF.

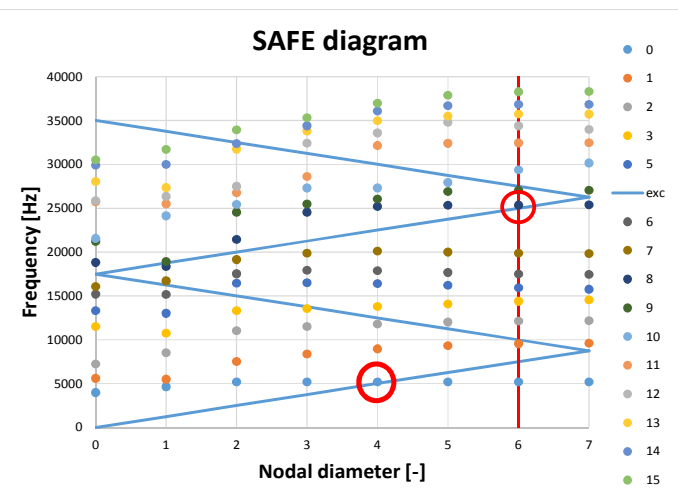

Figure 14: SAFE Diagram for the Original Impeller
The original and optimized overall performance are summarized in Table 2.

Table 2: Original and optimized overall performance for radial inflow turbine

\begin{tabular}{c||c|c} 
& Original & Optimised \\
\hline \hline$\dot{m}[\mathrm{~kg} / \mathrm{s}]$ & 0.907 & 0.75 \\
\hline$\eta_{t-t}[-]$ & 0.872 & 0.927 \\
\hline Power $[\mathrm{kW}]$ & 250.24 & 246.17 \\
\hline$u / c_{0}$ & 0.655 & 0.651 \\
\hline Stress usage factor $[-]$ & 2.54 & 0.98 \\
\hline Resonance 4OE & Yes & No \\
\hline Resonance NPF & Yes & No \\
\hline
\end{tabular}

By employing the recuperator described in the first part of the paper and by using the efficiencies achieved through the presented optimization design procedure, the resulting overall cycle efficiency raises from $30 \%$ to $36 \%$.

\section{Conclusions}

In this work a multidisciplinary optimization procedure, partially based on open source software, has been described and discussed.

The aim of the work was to illustrate a multidisciplinary procedure capable of increasing aerodynamic efficiency guaranteeing mechanical safety for high speed radial gas turbines and centrifugal compressors. The paper illustrates the coupling between a widely used CFD commercial software and open source tools for mesh generation and mechanical analysis. The coupling has been obtained via in-house developed Python and Fortran scripts suited for the current application.

Through this integrated design approach, starting from a preliminary 1D design, the following goals have been achieved:

- $\quad$ higher efficiency;

- Von Mises stress field below the allowable limits, defined as a function of temperature for the turbine case;

- free from resonance behavior of the two impellers, in the speed operating regime, for the sources of excitation considered.

- The efficiency enhancement achieved for the singular components enables to obtain an overall efficiency increase of around 6\%, bringing the state of the art overall efficiency from a starting value of $30 \%$ up to an optimized value of about $36 \%$, close to the reciprocating engines one. 


\section{Nomenclature}

ANN Multi Layer Perceptron Artificial Neura Network

a Constant variable

$b \quad$ Channel width $[m]$

$c \quad$ Absolute velocity $[\mathrm{m} / \mathrm{s}]$

CFD Computational Fluid Dynamics

$c_{p} \quad$ Pressure coefficient [dimensionless]

D Diameter $[m]$

DoE Design of Experiments

EO Engine Order

FEA Finite Element Analysis

$f \quad$ Frequency $[\mathrm{Hz}]$

GA Genetic algorithm

$L \quad$ Excitation harmonic number [dimensionless $]$

$L E \quad$ Leading Edge

$L_{1-2} \quad$ Specific work $[\mathrm{J} / \mathrm{kg}]$

$M \quad$ Nodal diameter [dimensionless]

$m$

$\dot{m}$

$M a$

$M D O$

$N_{\text {roto }}$

$N_{\text {vane }} \quad$ Number of vane blades

Shaft speed $[\mathrm{rpm}]$

$n_{s} \quad$ Specific speed based on machine outlet density [radians]

$\bar{n}_{s} \quad$ Specific speed based on impeller inlet density [radians]

$P \quad$ Penalty function

RANS Reynolds Averaged Navier Stokes Equations

SF Safety Factor [dimensionless]

$T \quad$ Temperature $[K]$

TE Trailing Edge

TIT Turbine inlet total temperature $[K]$
$W$

$w$

$y^{+}$

\section{Greeks}

$\alpha$

$\beta$

$\eta$

$\vartheta$

$\sigma$

\section{Subscripts}

0

1

2

Aero

all

eigfr

exc

Freq_high

Freq_low

$i$

is

MAX

$M I N$

$N P F$

OUT

$r$

$R O T$

Stress

Struct

$t-t$

$y$
Peripheral speed $[\mathrm{m} / \mathrm{s}]$

Weight term for penalty function [dimensionless]

Relative velocity $[\mathrm{m} / \mathrm{s}]$

Dimensionless wall distance [dimensionless]
Absolute flow angle [deg]

Pressure ratio [dimensionless]

Efficiency [dimensionless]

Tangential coordinate $[\mathrm{deg}]$

Stress $[\mathrm{MPa}]$
Inlet section

Interstage section

Outlet section

Aerodynamic

Allowable

Eigenfrequency

Exciting

High frequency

Low frequency

Generic index

Isentropic

Maximum

Minimum

Relative to nozzle passing frequency

Outlet

Relative

Rotation

Referred to stress analysis

Structural

Total to total

Yielding 


\section{References}

[1] Mueller, L., Alsalihi, Z., Verstraete, T., 2013, "Multidisciplinary Optimization ofa Turbocharger Radial Turbine", Journal of Turbomachinery, Volume 135

[2] Verstraete, T., Alsalihi, Z., Van den Braembussche R., 2010, "Multidisciplinary Optimization of a Radial Compressor for Microgas Turbine Applications", Journal of Turbomachinery, Volume 132

[3] Dario Barsi, Andrea Perrone, Luca Ratto, Daniele Simoni, Pietro Zunino, "Radial inflow turbine design through Multidisciplinary optimization technique", In Proceedings of ASME Turbo Expo 2015: Turbine Technical Conference and Exposition GT2015 June 15 - 19, 2015, Montreal, Canada.
[4] Vavra M. H., "Basic elements for advanced designs of radial flow compressors", AGARD Lecture Series No 39, 1970.

[5] NUMECA, User manuals. Academic R\&D license 2014.

[6] Hirsch C., Lacor C., Rizzi A., Eliasson P., Lindblad I. and Haeuser J., "A multiblock/multigrid code for the efficient solution of complex 3D Navier-Stokes flows", Aerothermodynamics for Space Vehicles 1, pp. 415-420, SEE N92-14973 06-02, 1991

[7] Geuzaine,C., Remacle, J. F., 2009,“Gmsh: a threedimensional finite element mesh generator with built-in pre- and post-processing facilities", International Journal for Numerical Methods in Engineering, Volume 79, Issue 11 , pages 1309-1331.

[8] www.calculix.de 